\title{
Prostate Cancer Risk Calculator Apps in a Taiwanese Population Cohort: Validation Study
}

I-Hsuan Alan Chen ${ }^{1,2,3,4}$, MSc, MD; Chi-Hsiang Chu ${ }^{5}$, PhD; Jen-Tai Lin ${ }^{1,2}, \mathrm{MD}, \mathrm{PhD}$; Jeng-Yu Tsai ${ }^{1,2}, \mathrm{MD}, \mathrm{PhD}$; Chia-Cheng Yu ${ }^{1,2,3}$, MD; Ashwin Narasimha Sridhar ${ }^{4,6}$, FRCS, MSc; Prasanna Sooriakumaran ${ }^{4,6,7}$, FRCS, PhD; Rui C V Loureiro ${ }^{4,8}$, PhD; Manish Chand ${ }^{4,9}$, MBA, FRCS, PhD

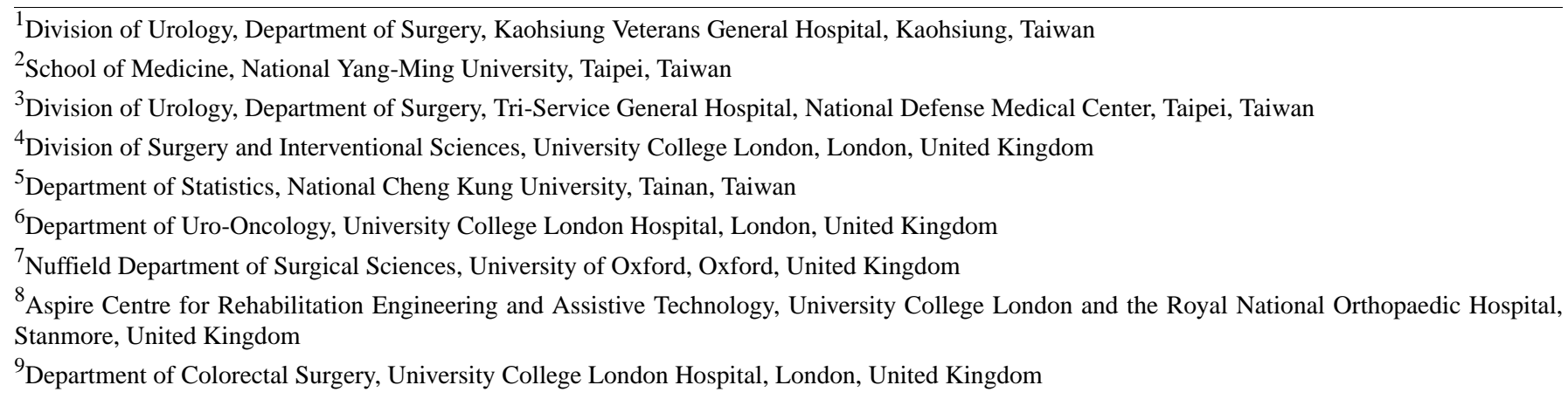

\section{Corresponding Author:}

Jen-Tai Lin, MD, PhD

Division of Urology

Department of Surgery

Kaohsiung Veterans General Hospital

No 386, Dazhong 1st Rd

Zuoying District

Kaohsiung, 813414

Taiwan

Phone: 886975939290

Email: jtlin@vghks.gov.tw

\begin{abstract}
Background: Mobile health apps have emerged as useful tools for patients and clinicians alike, sharing health information or assisting in clinical decision-making. Prostate cancer (PCa) risk calculator mobile apps have been introduced to assess risks of $\mathrm{PCa}$ and high-grade PCa (Gleason score $\geq 7$ ). The Rotterdam Prostate Cancer Risk Calculator and Coral-Prostate Cancer Nomogram Calculator apps were developed from the 2 most-studied PCa risk calculators, the European Randomized Study of Screening for Prostate Cancer (ERSPC) and the North American Prostate Cancer Prevention Trial (PCPT) risk calculators, respectively. A systematic review has indicated that the Rotterdam and Coral apps perform best during the prebiopsy stage. However, the epidemiology of PCa varies among different populations, and therefore, the applicability of these apps in a Taiwanese population needs to be evaluated. This study is the first to validate the PCa risk calculator apps with both biopsy and prostatectomy cohorts in Taiwan.
\end{abstract}

Objective: The study's objective is to validate the PCa risk calculator apps using a Taiwanese cohort of patients. Additionally, we aim to utilize postprostatectomy pathology outcomes to assess the accuracy of both apps with regard to high-grade PCa.

Methods: All male patients who had undergone transrectal ultrasound prostate biopsies in a single Taiwanese tertiary medical center from 2012 to 2018 were identified retrospectively. The probabilities of PCa and high-grade PCa were calculated utilizing the Rotterdam and Coral apps, and compared with biopsy and prostatectomy results. Calibration was graphically evaluated with the Hosmer-Lemeshow goodness-of-fit test. Discrimination was analyzed utilizing the area under the receiver operating characteristic curve (AUC). Decision curve analysis was performed for clinical utility.

Results: Of 1134 patients, 246 (21.7\%) were diagnosed with PCa; of these 246 patients, 155 (63\%) had high-grade PCa, according to the biopsy results. After confirmation with prostatectomy pathological outcomes, 47.2\% (25/53) of patients were upgraded to high-grade PCa, and 1.2\% (1/84) of patients were downgraded to low-grade PCa. Only the Rotterdam app demonstrated 
good calibration for detecting high-grade PCa in the biopsy cohort. The discriminative ability for both PCa (AUC: 0.779 vs 0.687; DeLong's method: $P<.001$ ) and high-grade PCa (AUC: 0.862 vs $0.758 ; P<.001$ ) was significantly better for the Rotterdam app. In the prostatectomy cohort, there was no significant difference between both apps (AUC: 0.857 vs $0.777 ; P=.128$ ).

Conclusions: The Rotterdam and Coral apps can be applied to the Taiwanese cohort with accuracy. The Rotterdam app outperformed the Coral app in the prediction of PCa and high-grade PCa. Despite the small size of the prostatectomy cohort, both apps, to some extent, demonstrated the predictive capacity for true high-grade PCa, confirmed by the whole prostate specimen. Following our external validation, the Rotterdam app might be a good alternative to help detect PCa and high-grade PCa for Taiwanese men.

(J Med Internet Res 2020;22(12):e16322) doi: $\underline{10.2196 / 16322}$

\section{KEYWORDS}

diagnosis; mHealth; mobile apps; prostate cancer; prostate-specific antigen; risk calculator

\section{Introduction}

The use of health-related apps is increasing within health care systems. Prostate cancer (PCa) risk calculator mobile apps have been introduced to assess risks of $\mathrm{PCa}$ and high-grade $\mathrm{PCa}$ (Gleason score $\geq 7$ ). The Rotterdam Prostate Cancer Risk Calculator and Coral-Prostate Cancer Nomogram Calculator apps were developed from the 2 most-studied PCa risk calculators, the European Randomized Study of Screening for Prostate Cancer (ERSPC) [1] and the North American Prostate Cancer Prevention Trial (PCPT) [2] risk calculators, respectively. Adam et al [3] performed a critical appraisal of 7 PCa risk calculator apps, indicating that the Rotterdam and Coral apps performed best during the prebiopsy stage. According to the currently available evidence, both apps have only been externally validated by a 2-center European study. They have demonstrated better predictive accuracy than prostate-specific antigen (PSA) and digital rectal examination (DRE) [4].

In Taiwan, around $40 \%$ of new PCa cases are diagnosed as locally advanced or metastatic diseases, which is less favorable than the stage distribution of Western countries [5]. This has not changed remarkably over the last two decades, albeit the incidence of PCa has been increasing in Taiwan since 1979. Metastatic PCa still made up almost $30 \%$ of newly-diagnosed cases from 2004 to 2012 compared to a proportion of $32.7 \%$ from 1977 to 1997 [6]. For early detection of PCa, risk calculator apps may help assess the risk of PCa or high-grade PCa in the Taiwanese population. Moreover, with the capacity to differentiate high-grade PCa, active surveillance might be supported by these apps during patient counseling.

The aim of this study is to evaluate the performance of PCa risk calculator apps in a Taiwanese population. We performed external validation using a Taiwanese cohort of patients who had undergone transrectal ultrasound (TRUS) prostate biopsy. Additionally, in previous validation studies for $\mathrm{PCa}$ risk calculators or apps, risk stratification was based on biopsy outcomes instead of postprostatectomy pathology results. Accordingly, we aimed to utilize postprostatectomy pathology outcomes to assess the accuracy of both apps with regard to high-grade $\mathrm{PCa}$.

\section{Methods}

\section{Inclusion Criteria}

Internal review board approval (IRB No.: VGHKS19-CT3-13) was granted by a Taiwanese tertiary medical center, the Kaohsiung Veterans General Hospital. All male patients $(\mathrm{N}=1344)$ undergoing TRUS prostate biopsies with a 12-core systematic biopsy strategy from 2012 to 2018 were enrolled. The indication for prostate biopsy included an abnormal PSA level (>4 ng/mL) or an abnormal DRE. Each patient would receive DRE and TRUS before the biopsy was performed; prostate volume (PV) was calculated by the ellipsoid formula (length $\mathrm{x}$ width $\mathrm{x}$ height $\mathrm{x} \pi / 6)$. Some patients (53/1344) had multiparametric magnetic resonance imaging (mpMRI) scans on a self-pay basis because the Taiwan National Health Insurance system has not approved the reimbursement of pelvic magnetic resonance imaging (MRI) before prostate biopsy. All prebiopsy mpMRI scans were reported by dedicated urologic radiologists, in agreement with the Prostate Imaging Reporting and Data System, version 2 (PI-RADS v2) [7]. The number of patients who received radical prostatectomy was 137 . Consultant pathologists reviewed all biopsies and postprostatectomy specimens.

According to the Rotterdam app, the definition of clinically significant $\mathrm{PCa}$ is a tumor stage greater than $\mathrm{T} 2 \mathrm{~b}$, or a Gleason biopsy score of $\geq 7$, which is identical to high-risk $\mathrm{PCa}$ in the ERSPC risk calculator (ERSPC-RC) [8]. In comparison, the Coral app defined a Gleason biopsy score of $\geq 7$ as high-grade $\mathrm{PCa}$, which originated from the PCPT [2]. In order to use consistent terminology, a Gleason score of $\geq 7$ was defined as high-grade PCa. PSA was designated as the latest total serum PSA level before prostate biopsy.

\section{Data Collection}

All patient data were retrospectively collected via electronic medical records. The Rotterdam app accepts input data on age, DRE history and outcome, previous negative biopsy, PSA, PV, volume measure method (TRUS or DRE), TRUS evaluation (normal or abnormal), MRI history, and PI-RADS score. The Coral app requires data on ethnicity (African American, Caucasian, Hispanic, or Other), age, DRE, PSA, family history, and prior biopsy results. Following input data collection, the risks of PCa and high-grade $\mathrm{PCa}$ were calculated using the Rotterdam and Coral apps. 


\section{Exclusion Criteria}

Each app customizes its parameters to impose controls or constraints on accepted input values; that is, both the Rotterdam and Coral apps have an input data range for some parameters. For instance, the input PSA range is limited from $0.4-50 \mathrm{ng} / \mathrm{mL}$ within the Rotterdam app and $0.3-100 \mathrm{ng} / \mathrm{mL}$ within the Coral app. If the patients have prebiopsy MRI scans, there is an age limit between 50 and 75 years. On the contrary, without prebiopsy MRI, both the Rotterdam and Coral apps have no limitation on the input age data. According to the accepted input values from both apps, 197 patients were excluded, either because their PV was $<10$ or $>110 \mathrm{~mL}(68 / 197)$, or their PSA level was $<0.4$ or $>50 \mathrm{ng} / \mathrm{mL}(118 / 197)$, or they underwent prebiopsy MRI at the age of $<50$ or $>75$ years $(11 / 197)$. In addition, 13 more patients with previous positive biopsies (8/13), pathological diagnosis different from adenocarcinoma (3/13), or incomplete data (2/13) were excluded. Details of the inclusion and exclusion process are illustrated in the flow chart in Figure 1 .

Figure 1. Flowchart of patient enrollment into the study. MRI: magnetic resonance imaging; PSA: prostate-specific antigen; TRUS: transrectal ultrasound.

Inclusion

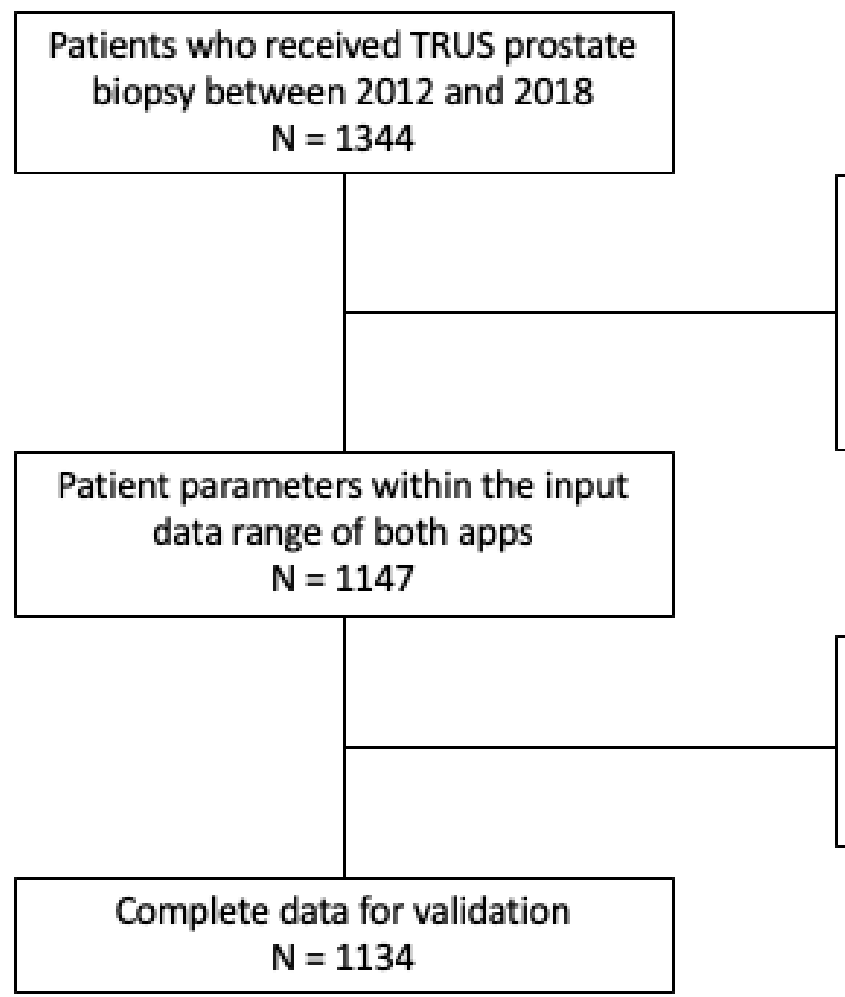

\section{Exclusion}

Patient parameters out of the input data range of both apps:

Age $>75$ receiving pre-biopsy MRI $(\mathrm{N}=11)$

Prostate Volume $>110 \mathrm{~mL}(\mathrm{~N}=68)$ $\mathrm{PSA}>50 \mathrm{ng} / \mathrm{mL}(\mathrm{N}=118)$

\section{Previous positive biopsy $(\mathrm{N}=8)$ \\ Biopsy pathological result other than adenocarcinoma $(\mathrm{N}=3)$ Incomplete data $(\mathrm{N}=2)$}

\section{Statistical Analysis}

Statistical analyses were performed utilizing SPSS (version 18; IBM Corp) and $\mathrm{R}$ software packages (R Core Team). The Kolmogorov-Smirnov test was used to examine the normality of the distribution of variables. Categorical variables were assessed with the chi-square test. Continuous variables were described as medians and interquartile ranges or means and standard deviations, and compared by the Mann-Whitney $U$ test or the Student independent $t$ test based upon their nonnormal or normal distributions, respectively. The applicability of each PCa risk calculator app in the Taiwanese population cohort was statistically analyzed on the basis of its discrimination, calibration, and clinical utility [9]. Calibration relates to the agreement between the observed and predicted proportion of events; calibration was evaluated graphically utilizing a calibration plot in which the observed probabilities were plotted against the predicted probabilities, enabling assessment of the extent of risk underestimation or overestimation [10]. The statistical significance of miscalibration was examined by the Hosmer-Lemeshow goodness-of-fit test [11].

Discrimination reflects the capacity of a prediction model to differentiate between those with and without an event (any-grade or high-grade $\mathrm{PCa}$ ) and is quantified utilizing the area under the receiver operating characteristic (ROC) curve (AUC). The AUCs of the Rotterdam and Coral apps were compared using DeLong's method [12]. As for clinical utility, decision curve analysis was performed to analyze whether both apps were beneficial for clinical decision-making or which app would lead to better decisions. We calculated the net benefit to quantify the clinical utility; different threshold probabilities mean different harm-to-benefit ratios. Net benefit was formulated as the number of true positives subtracted from the proportion of false positives weighted by the odds of the risk threshold probability, and the result was divided by the sample size. By 
measuring the proportions of net true positives in the models, we could assess whether any model performed better than others and the default strategies of biopsying all or no patients across the reasonable range of risk threshold probabilities [13].

\section{Results}

\section{Patient Demographics}

Of 1344 patients undergoing biopsies, 246 (21.7\%) patients were diagnosed with $\mathrm{PCa}$; of these 246 patients, 155 (63\%) had high-grade $\mathrm{PCa}$, according to the biopsy results. Compared to males with negative biopsies, patients with $\mathrm{PCa}$ were significantly older, had higher PSA levels, smaller PVs, more abnormal findings on TRUS and DRE, and higher PI-RADS scores demonstrated on mpMRI (Table 1). Both in the biopsy and prostatectomy cohorts, patients with high-grade PCa had significantly higher PSA, lower PV, and more abnormal findings on TRUS compared to those with low-grade PCa (Table 2). Among 246 diagnoses of PCa, 137 patients underwent radical prostatectomy; based on the postprostatectomy outcomes, $47.2 \%$ $(25 / 53)$ of patients were upgraded to high-grade $\mathrm{PCa}$ and $1.2 \%$ (1/84) of patients were downgraded to low-grade PCa.

Table 1. Patient demographics $(\mathrm{N}=1344)$. Categorical variables were assessed with the chi-square test; continuous variables were compared by the Mann-Whitney $\mathrm{U}$ test or the Student independent $\mathrm{t}$ test based on their nonnormal or normal distribution, respectively.

\begin{tabular}{|c|c|c|c|c|}
\hline Characteristics & All patients $(\mathrm{n}=1134)$ & $\begin{array}{l}\text { Patients with no cancer }(\mathrm{n}=888 \text {, } \\
78.3 \%)\end{array}$ & $\begin{array}{l}\text { Patients with cancer }(n=246, \\
21.7 \%)\end{array}$ & $P$ value \\
\hline $\begin{array}{l}\text { Age in years, mean (SD); median ( } 1^{\text {st }} \\
\left.\text { quartile- } 3^{\text {rd }} \text { quartile }\right)\end{array}$ & 68.78 (8.23); 67 (61-73) & 66.31 (8.17); 66 (61-72) & $68.49(8.25) ; 69$ (63-74) & $<.001$ \\
\hline $\begin{array}{l}\mathrm{PSA}^{\mathrm{a}} \text {, mean }(\mathrm{SD}) \text {; median ( } 1^{\text {st }} \text { quar- } \\
\left.\text { tile- } 3^{\text {rd }} \text { quartile }\right)\end{array}$ & $10.27(7.44) ; 8.08(5.73-11.65)$ & $9.20(5.90) ; 7.66(5.57-10.44)$ & $\begin{array}{l}14.12(10.53) ; \\
10.16(6.64-17.51)\end{array}$ & $<.001$ \\
\hline $\begin{array}{l}\mathrm{PV}^{\mathrm{b}} \text {, mean }(\mathrm{SD}) ; \text { median }\left(1^{\text {st }} \text { quartile- }\right. \\
\left.3^{\text {rd }} \text { quartile }\right)\end{array}$ & $\begin{array}{l}52.72(21.13) ; 48.35(37.08- \\
66.01)\end{array}$ & $\begin{array}{l}55.35(20.86) ; 51.52(39.93- \\
68.88)\end{array}$ & $\begin{array}{l}43.25(19.36) \\
37.82(29.68-52.00)\end{array}$ & $<.001$ \\
\hline Family history, n (\%) & $44(3.9)$ & $32(3.6)$ & $12(4.9)$ & .360 \\
\hline Suspicious TRUS ${ }^{\mathrm{c}}, \mathrm{n}(\%)$ & $253(22.3)$ & $151(17.0)$ & $102(41.5)$ & $<.001$ \\
\hline Suspicious DRE ${ }^{\mathrm{d}}, \mathrm{n}(\%)$ & $192(16.9)$ & $89(10.0)$ & $103(41.9)$ & $<.001$ \\
\hline $\operatorname{MRI}^{\mathrm{e}}(\mathrm{n}=38)$ PI-RADS ${ }^{\mathrm{f}} 4,5, \mathrm{n}(\%)$ & $27(71.1)$ & $14(58.3)$ & $13(92.9)$ & .030 \\
\hline $\begin{array}{l}\text { Rotterdam PCa } \mathrm{P}^{\mathrm{g}}(\%) \text {, mean }(\mathrm{SD}) ; \text { me- } \\
\text { dian }\left(1^{\text {st }} \text { quartile- } 3^{\text {rd }} \text { quartile }\right)\end{array}$ & 29.98 (23.98); 21 (13-39) & 23.92 (17.69); $18(12-31)$ & $51.84(30.20) ; 50(24-84)$ & $<.001$ \\
\hline $\begin{array}{l}\text { Rotterdam high-grade } \mathrm{PCa}(\%) \text {, mean } \\
\text { (SD); median }\left(1^{\text {st }} \text { quartile- } 3^{\text {rd }} \text { quar- }\right. \\
\text { tile) }\end{array}$ & $14.30(21.47) ; 5(2-14)$ & $8.66(12.64) ; 4(2-9)$ & $34.60(31.98) ; 21(6-65)$ & $<.001$ \\
\hline $\begin{array}{l}\text { Coral PCa }(\%) \text {, mean }(\mathrm{SD}) \text {; median } \\
\left(1^{\text {st }} \text { quartile- } 3^{\text {rd }} \text { quartile }\right)\end{array}$ & $34.37(11.53) ; 32(26-39)$ & $32.42(9.64) ; 31(26-36)$ & $41.42(14.63) ; 38(31.00-51.25)$ & $<.001$ \\
\hline $\begin{array}{l}\text { Coral high-grade } \mathrm{PCa}(\%) \text {, mean } \\
(\mathrm{SD}) \text {; median }\left(1^{\text {st }} \text { quartile- } 3^{\text {rd }} \text { quar- }\right. \\
\text { tile) }\end{array}$ & $14.59(10.38) ; 11(8-18)$ & $\begin{array}{l}12.74(8.05) \\
11(7-16)\end{array}$ & $21.24(14.38) ; 17(10-30)$ & $<.001$ \\
\hline
\end{tabular}

${ }^{a}$ PSA: prostate-specific antigen.

${ }^{\mathrm{b}} \mathrm{PV}$ : prostate volume.

${ }^{\mathrm{c}}$ TRUS: transrectal ultrasound.

${ }^{\mathrm{d} D R E}$ : digital rectal examination.

${ }^{\mathrm{e}}$ MRI: magnetic resonance imaging.

fPI-RADS: Prostate Imaging Reporting and Data System.

${ }^{\mathrm{g}} \mathrm{PCa}$ : prostate cancer. 
Table 2. Demographics of patients with prostate cancer ( $\mathrm{PCa} ; \mathrm{n}=383$ ). Categorical variables were assessed with the chi-square test; continuous variables were compared by the Mann-Whitney U test or the Student independent t test based on their nonnormal or normal distribution, respectively.

\begin{tabular}{|c|c|c|c|c|c|c|}
\hline \multirow[t]{2}{*}{ Characteristics } & \multicolumn{3}{|c|}{ Biopsy cohort $(n=246)$} & \multicolumn{3}{|c|}{ Prostatectomy cohort $(n=137)$} \\
\hline & $\begin{array}{l}\text { Low-grade } \mathrm{PCa}^{\mathrm{a}} \\
(\mathrm{n}=91,37 \%)\end{array}$ & $\begin{array}{l}\text { High-grade PCa } \\
(\mathrm{n}=155,63 \%)\end{array}$ & $P$ value & $\begin{array}{l}\text { Low-grade PCa } \\
(\mathrm{n}=29,21 \%)\end{array}$ & $\begin{array}{l}\text { High-grade PCa } \\
(\mathrm{n}=108,79 \%)\end{array}$ & $P$ value \\
\hline Age in years, mean (SD) & $67.22(8.00)$ & $69.24(8.33)$ & .064 & $63.07(7.21)$ & $65.79(6.27)$ & .047 \\
\hline $\begin{array}{l}\text { PSA }^{b} \text {, mean }(S D) ; \text { median }\left(1^{\text {st }} \text { quartile- }\right. \\
\left.3^{\text {rd }} \text { quartile }\right)\end{array}$ & $\begin{array}{l}9.94(7.36) ; 7.9 \\
(5.1-12.2)\end{array}$ & $\begin{array}{l}16.57(11.33) ; 13.0 \\
(8.1-23.2)\end{array}$ & $<.001$ & $\begin{array}{l}6.68(2.60) \\
7.1(4.7-8.4)\end{array}$ & $\begin{array}{l}14.33(10.27) \\
11.1(6.7-17.9)\end{array}$ & $<.001$ \\
\hline $\begin{array}{l}\mathrm{PV}^{\mathrm{c}} \text {, mean }(\mathrm{SD}) ; \text { median }\left(1^{\text {st }} \text { quartile- }\right. \\
\left.3^{\text {rd }} \text { quartile }\right)\end{array}$ & $\begin{array}{l}47.56(22.30) ; 42.4 \\
(30.4-59.7)\end{array}$ & $\begin{array}{l}40.71(16.97) ; 36.4 \\
(28.9-48.9)\end{array}$ & .039 & $\begin{array}{l}51.53(22.13) ; 46.6 \\
(32.5-61.0)\end{array}$ & $\begin{array}{l}39.80(17.26) ; 34.2 \\
(28.6-46.3)\end{array}$ & .004 \\
\hline Family history, n (\%) & $4(4.4)$ & $8(5.2)$ & $>.99$ & $1(3.4)$ & $7(6.5)$ & $>.99$ \\
\hline Suspicious TRUS $^{\mathrm{d}}, \mathrm{n}(\%)$ & $18(19.8)$ & $84(54.2)$ & $<.001$ & $1(3.4)$ & $41(38.0)$ & $<.001$ \\
\hline Suspicious DRE ${ }^{\mathrm{e}}, \mathrm{n}(\%)$ & $27(29.7)$ & $76(49.0)$ & .003 & $7(24.1)$ & $45(41.7)$ & .084 \\
\hline MRI $^{\mathrm{f}}(\mathrm{n}=14) \mathrm{PI}^{-R_{A D S}}{ }^{\mathrm{g}} 4,5, \mathrm{n}(\%)$ & $2(100.0)$ & $11(91.7)$ & $>.99$ & - & $10(90.9)$ & - \\
\hline $\begin{array}{l}\text { Rotterdam PCa (\%), mean (SD); medi- } \\
\text { an }\left(1^{\text {st }} \text { quartile- } 3^{\text {rd }} \text { quartile }\right)\end{array}$ & $\begin{array}{l}35.13(26.39) ; 25.0 \\
(15.0-54.0)\end{array}$ & $\begin{array}{l}61.65(27.96) \\
66.0(36.0-88.0)\end{array}$ & $<.001$ & $\begin{array}{l}21.72(14.70) \\
18.0(12.5-25.0)\end{array}$ & $\begin{array}{l}54.32(27.91) \\
52.0(28.3-84.0)\end{array}$ & $<.001$ \\
\hline $\begin{array}{l}\text { Rotterdam high-grade PCa }(\%) \text {, mean } \\
(\mathrm{SD}) ; \text { median }\left(1^{\text {st }} \text { quartile- } 3^{\text {rd }} \text { quartile }\right)\end{array}$ & $\begin{array}{l}18.74(25.72) \\
7.0(3.0-21.0)\end{array}$ & $\begin{array}{l}43.92(31.69) \\
36.0(15.0-75.0)\end{array}$ & $<.001$ & $\begin{array}{l}7.14(8.50) \\
4.0(3.0-7.0)\end{array}$ & $\begin{array}{l}34.39(28.93) \\
23.5(10.0-60.3)\end{array}$ & $<.001$ \\
\hline $\begin{array}{l}\text { Coral PCa }(\%) \text {, mean }(\mathrm{SD}) ; \text { median }\left(1^{\text {st }}\right. \\
\left.\text { quartile- } 3^{\text {rd }} \text { quartile }\right)\end{array}$ & $\begin{array}{l}35.11(11.86) \\
31.0(26.0-41.0)\end{array}$ & $\begin{array}{l}45.13(14.86) \\
42.0(34.0-55.0)\end{array}$ & $<.001$ & $\begin{array}{l}28.34(6.14) \\
27.0(24.0-34.0)\end{array}$ & $\begin{array}{l}39.43(12.29) \\
37.0(30.0-48.0)\end{array}$ & $<.001$ \\
\hline $\begin{array}{l}\text { Coral high-grade } \mathrm{PCa}(\%) \text {, mean }(\mathrm{SD}) \text {; } \\
\text { median }\left(1^{\text {st }} \text { quartile- } 3^{\text {rd }} \text { quartile }\right)\end{array}$ & $\begin{array}{l}15.37(10.71) \\
12.0(8.0-19.0)\end{array}$ & $\begin{array}{l}24.68(15.16) \\
20.0(13.0-34.0)\end{array}$ & $<.001$ & $\begin{array}{l}9.48(4.99) \\
8.0(6.0-13.5)\end{array}$ & $\begin{array}{l}18.92(11.55) \\
15.0(10.0-25.0)\end{array}$ & $<.001$ \\
\hline
\end{tabular}

${ }^{\mathrm{a}} \mathrm{PCa}$ : prostate cancer.

${ }^{\mathrm{b}}$ PSA: prostate-specific antigen.

${ }^{\mathrm{c}} \mathrm{PV}$ : prostate volume.

${ }^{\mathrm{d}}$ TRUS: transrectal ultrasound.

${ }^{\mathrm{e}}$ DRE: digital rectal examination.

f MRI: magnetic resonance imaging.

g PI-RADS: Prostate Imaging Reporting and Data System.

\section{Calibration}

The calibration of both apps was tested with the Hosmer-Lemeshow goodness-of-fit test (Figure 2). Comparing both apps, only the Rotterdam app demonstrated a good calibration $(P=.619)$ for detecting high-grade $\mathrm{PCa}$ in the biopsy cohort. Other models were miscalibrated, including all models created from the Coral app. 
Figure 2. Calibration plots comparing (a) the Rotterdam app and (b) Coral app showing the agreement between (i) predicted and observed probabilities for diagnosing prostate cancer (PCa) and (ii) high-grade PCa in the biopsy cohort, and (iii) high-grade PCa in the prostatectomy cohort. Each circle in the plots represents a group of patients with an observed probability of $\mathrm{PCa}$ or high-grade $\mathrm{PCa}$ on the $\mathrm{x}$-axis, corresponding to an average calculated risk of PCa or high-grade PCa by the apps on the y-axis. Figures (ia) and (ib) demonstrated overestimation, whereas Figures (iiia) and (iiib) illustrated underestimation. Only Figure (iia) showed a good calibration; in Figure (iib), overestimation was revealed among the lower observed proportions and underestimation among the higher observed proportions.

\section{PCa vs no PCa (biopsy cohort)}

ia

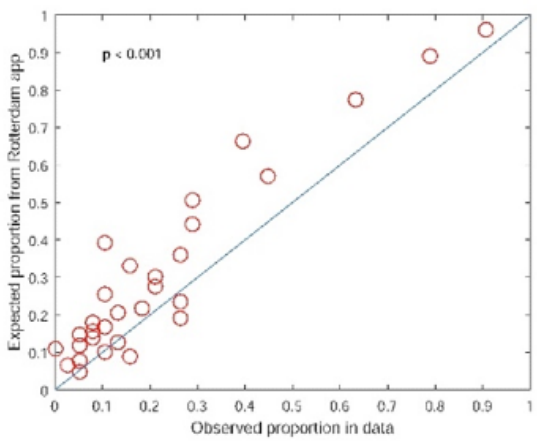

$\mathrm{ib}$

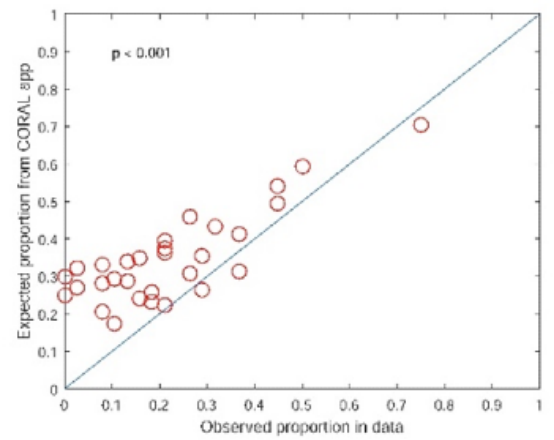

\section{High-grade PCa vs Low-grade and no PCa (biopsy cohort)}

iia

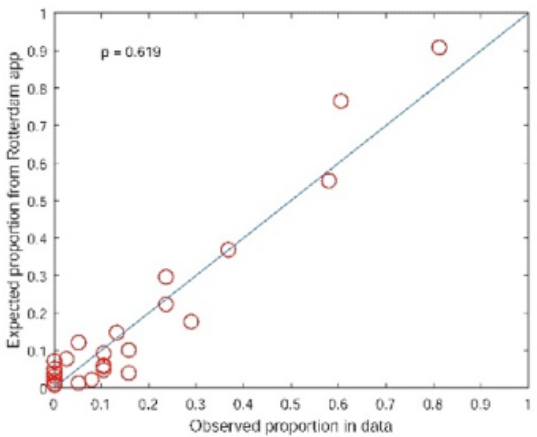

iib

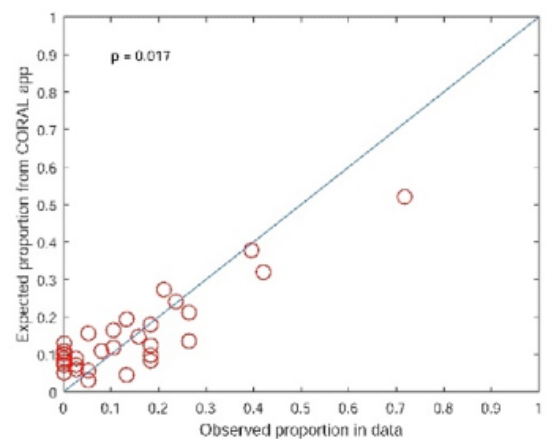

\section{High-grade PCa vs Low-grade PCa (prostatectomy cohort)}

iiia

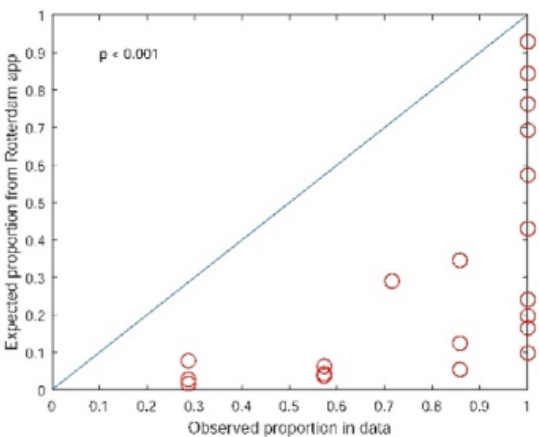

\section{Discrimination}

Both the Rotterdam and Coral apps could significantly predict $\mathrm{PCa}$ and high-grade $\mathrm{PCa}$ in the biopsy cohort on ROC analysis (Figure 3, a and b). The discriminative capacity for detection of both PCa (AUC: 0.779 vs 0.687; DeLong's method: $P<.001$ ) iiib

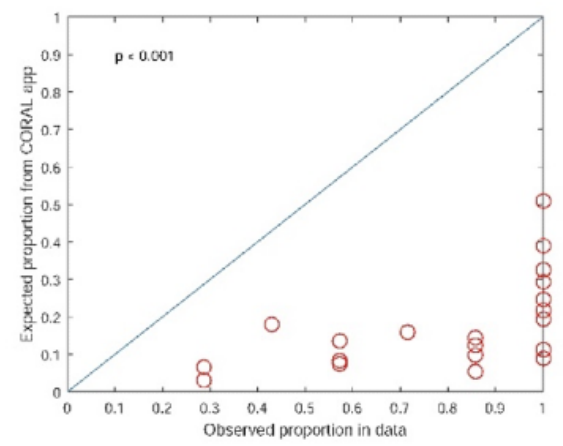

and high-grade PCa (AUC: 0.862 vs $0.758 ; P<.001$ ) was significantly better for the Rotterdam app compared to the Coral app. In the prostatectomy cohort, the Rotterdam and Coral apps were not significantly different for predicting high-grade $\mathrm{PCa}$ (AUC: 0.857 vs $0.777 ; P=.128$; Figure $3 \mathrm{c}$ ). 
Figure 3. Receiver operating characteristic (ROC) curves and areas under the receiver operating characteristic curves (AUCs) for the discriminative ability of the Rotterdam and Coral apps. (a) Prostate cancer (PCa) vs no PCa in the biopsy cohort; (b) high-grade PCa vs low-grade PCa plus no PCa in the biopsy cohort; (c) high-grade PCa vs low-grade PCa in the prostatectomy cohort.

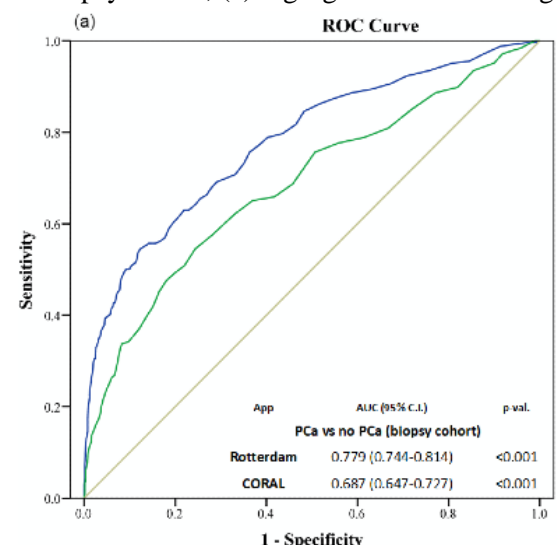

\section{Clinical Utility}

In the decision curve analysis for the biopsy cohort of patients, both apps demonstrated clinical net benefits in the threshold probability range of $10 \%$ to $85 \%$ for the detection of any PCa. In the detection of high-grade $\mathrm{PCa}$, the Rotterdam and Coral apps provided net benefits in the threshold probability range of 5\%-70\% and 10\%-80\%, respectively. In comparing both apps, the net benefit was greater for the Rotterdam app in the prediction of both $\mathrm{PCa}$ and high-grade $\mathrm{PCa}$ across the range of ROC Curve

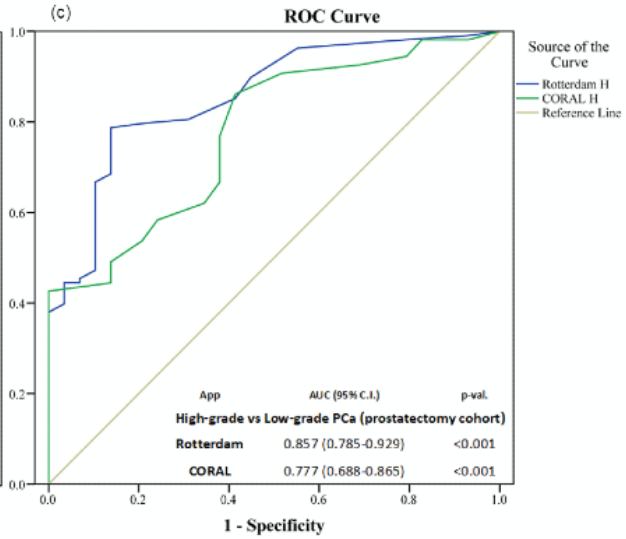

threshold probabilities from 5\%-70\% (Figure 4). It seemed that both apps provided net benefits for the detection of high-grade PCa in the prostatectomy cohort (Figure 5). Nevertheless, the prevalence of high-grade PCa in the prostatectomy cohort was very high (79\%). With baseline risk being very high, it would be difficult for both apps to push the risk to a level low enough for advice against biopsy. Both apps had higher net benefits when the curves diverged at the threshold probability of about $50 \%$, and therefore, both apps lacked value for the prediction of high-grade PCa in the prostatectomy cohort. 
Figure 4. Decision curve analyses quantifying clinical utility by showing the net benefits associated with the use of the Rotterdam app (blue line) and the Coral app (red line) in (a) the detection of prostate cancer (PCa) and (b) high-grade PCa. Decision curves investigate the theoretical net benefit at various threshold probabilities. The oblique gray line assumes that all persons will undergo prostate biopsy, whereas the horizontal black line along the $\mathrm{x}$-axis assumes that no one will receive biopsy. The threshold probability may correspond to the calculated prostate cancer risk. The area under the curve between these 2 lines illustrates net benefits. In the same range of threshold probability, higher net benefits represent better clinical utility.

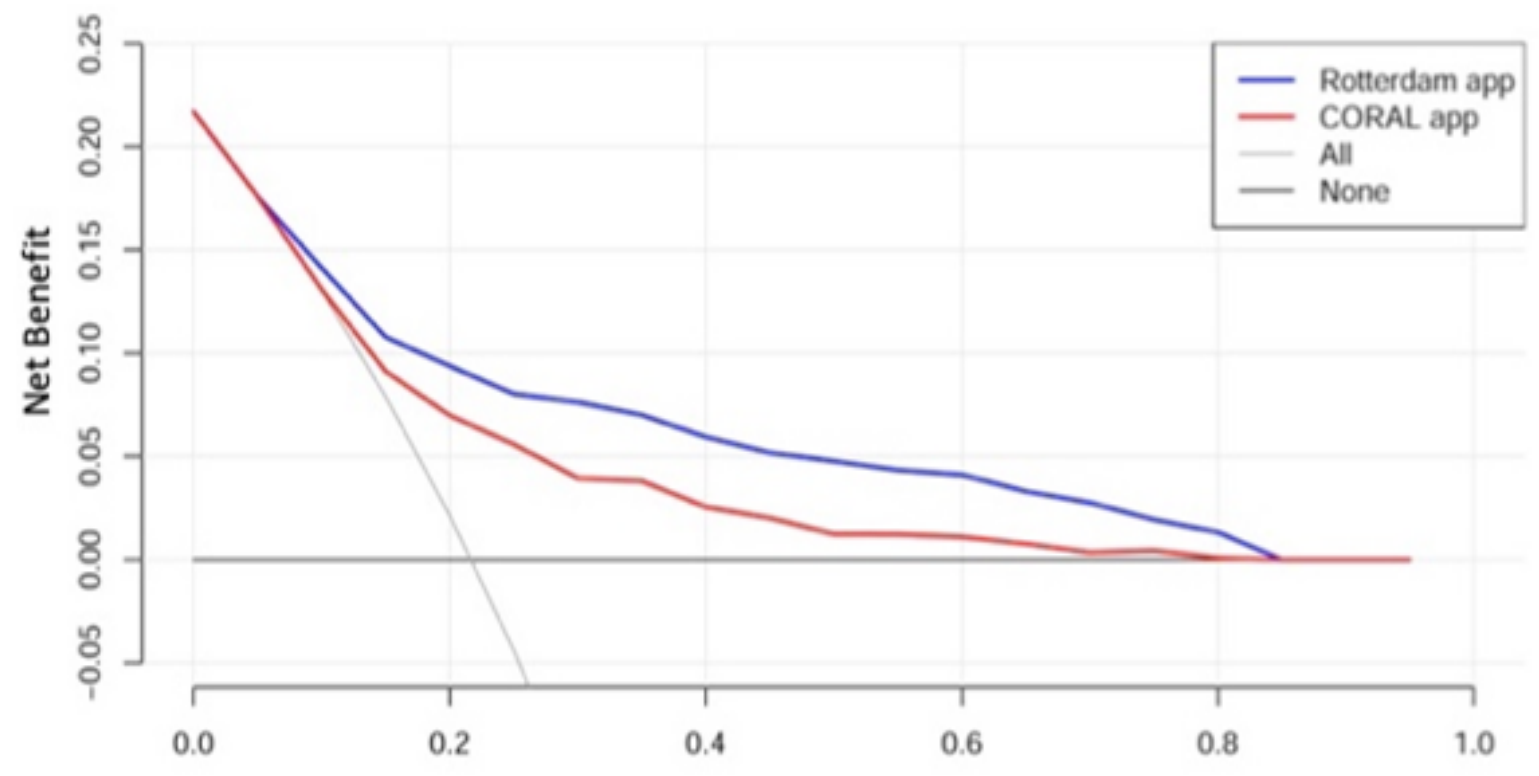

Threshold probability (\%)

a

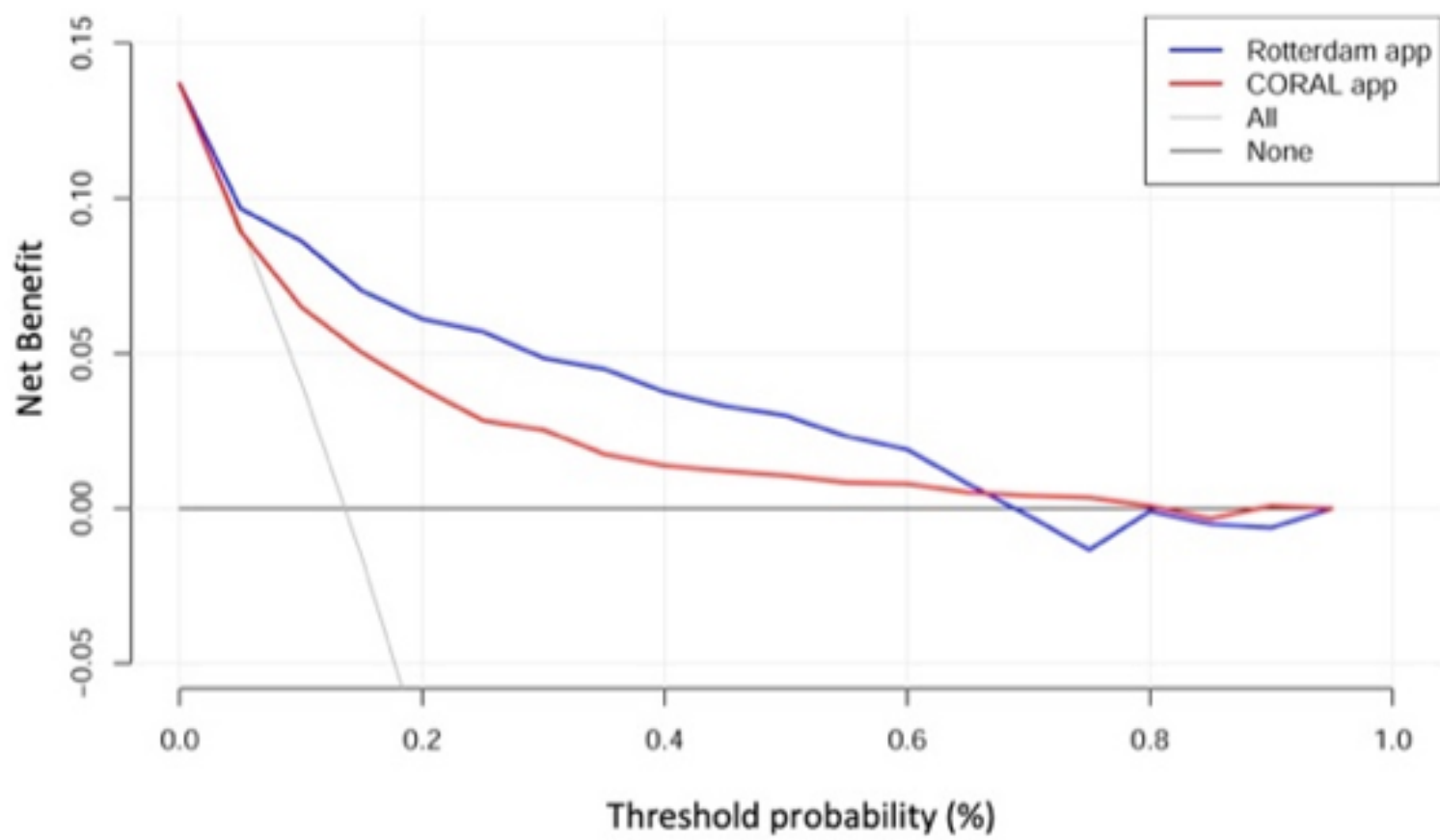

b 
Figure 5. Decision curve analysis showing the net benefits of utilizing the Rotterdam app (blue line) and the Coral app (red line) to detect high-grade prostate cancer ( $\mathrm{PCa}$ ) in the prostatectomy cohort. The curves are skewed because the incidence of high-grade PCa in the prostatectomy cohort is relatively high and the sample size is small. No net benefit could be demonstrated below the risk threshold of $50 \%$.

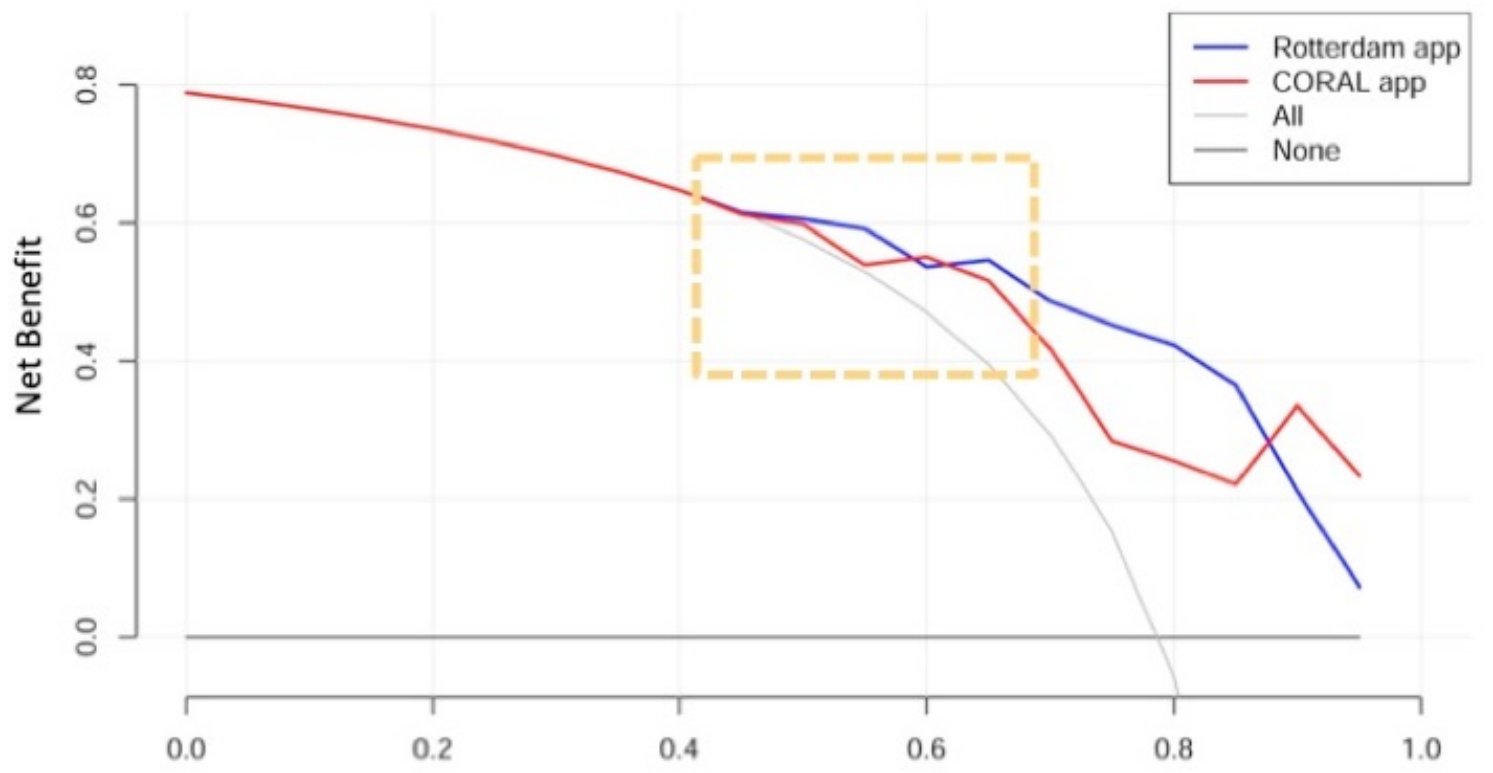

Threshold probability (\%)

\section{Discussion}

\section{Principal Findings}

In this study, we found that the Rotterdam and Coral apps were both applicable to the Taiwanese cohort of patients who had undergone TRUS prostate biopsy, even though these apps were built based on Western populations. In order to externally validate these 2 apps, 3 key statistical measures were used in the assessment of predictive performance. Firstly, most models revealed miscalibration, but the Rotterdam app demonstrated good calibration for the prediction of high-grade $\mathrm{PCa}$ in the biopsy cohort. Secondly, the Rotterdam app outperformed the Coral app in its discriminative ability for predicting PCa and high-grade PCa in the biopsy cohort. Thirdly, the Rotterdam app provided greater net benefits than the Coral app to assist in biopsy decision-making. In brief, the Rotterdam app delivered better predictive performance than the Coral app for $\mathrm{PCa}$ and high-grade PCa in our Taiwanese population cohort.

To the best of our knowledge, at the stage before PCa is diagnosed, no risk prediction model has ever been validated by the whole prostate specimen. Data from the 137 patients who had undergone radical prostatectomy for any-grade PCa were utilized to evaluate the Rotterdam and Coral apps' predictive capacity for high-grade PCa. Both apps demonstrated fairly good discrimination for predicting high-grade $\mathrm{PCa}$ in the prostatectomy cohort and the biopsy cohort. It was implied that after confirmation with postprostatectomy pathology outcomes, both apps still delivered a comparable discriminative ability for predicting high-grade $\mathrm{PCa}$ in the present Taiwanese cohort. However, during calibration and decision curve analysis in the prostatectomy cohort, both apps were miscalibrated and revealed few net benefits. This might be explained by the small sample size and different pathology distribution, as there was a higher prevalence of high-grade PCa $(79 \%)$. Moreover, these apps were built on biopsy cohorts, which are different from the prostatectomy cohort. More patients with radical prostatectomy might need to be enrolled to validate app predictability of high-grade $\mathrm{PCa}$, which would be validated by the whole prostate specimen.

In current clinical practice, most patients with an abnormal PSA level of $>4 \mathrm{ng} / \mathrm{mL}$ or an abnormal DRE are put forward for biopsy. However, such indications lead to a myriad of unnecessary biopsies and associated complications, such as hematuria, hematospermia, rectal bleeding, acute urinary retention, urinary tract infection, or even sepsis. To increase the accuracy of cancer detection and reduce unnecessary postbiopsy morbidities, several biomarker tests have been developed, including the Prostate Health Index (PHI), percent-free PSA, PCA3, 4K-score, etc [14]. The European Association of Urology (EAU) guidelines suggest an individualized evaluation of $\mathrm{PCa}$ risk. Age, family history of PCa, DRE, serum or urine markers, and mpMRI are validated parameters in combination with PSA levels to help predict the risk of PCa [15]. Recently, professionals have formulated a number of PCa risk calculators using some of these useful predictors to improve predictive accuracy, and such multivariable risk approaches have performed better than PSA or DRE alone [10]. Nonetheless, most of them have only been validated in independent cohorts; neither superiority nor global applicability has been shown [16]. De Nunzio et al [4] had validated the Rotterdam and Coral apps' discriminative abilities utilizing a southern European cohort as providing better predictive performance than PSA or DRE; however, the predictability of PCa or high-grade PCa in the Taiwanese population stills needs to be addressed.

It is well known that Gleason upgrading occurs in $32 \%-49 \%$ of patients with initial biopsy of low-grade (Gleason 3+3) PCa at the time of pathological assessment of the whole prostate specimen [14]. Verep et al [17] reviewed 137 patients who were 
eligible for active surveillance but underwent radical prostatectomy at their institution. The criteria of active surveillance included Gleason $3+3$ adenocarcinoma, maximum 2 positive biopsy cores, PSA $<10 \mathrm{ng} / \mathrm{mL}$, and clinical T-stage equal or less than $2 \mathrm{a}$. Following pathological confirmation, Gleason upgrading was noted in almost half of the patients (49.3\%), and upstaging to pT3a occurred in 17 patients $(12.5 \%)$ [17]. Due to the risks of over-diagnosis and over-treatment for clinically insignificant $\mathrm{PCa}$, active surveillance has become increasingly adopted as a preferred treatment option for patients with low-grade PCa. However, without precise risk stratification, active surveillance might delay the timing of curative treatment for localized $\mathrm{PCa}$, or even increase the risks of lymph node involvement and distant metastasis. Consequently, the accuracy of risk prediction tools has become of paramount importance.

Mobile health (mHealth) is regarded as a valuable tool to implement patient-centered care, which is in accordance with the individualized risk assessment of $\mathrm{PCa}$ recommended by the EAU guidelines. mHealth can provide access to health information, skills, and services, and can also promote positive health behavioral changes to prevent acute and chronic diseases. Real-time monitoring can obtain live data from patients and transmit inputs to a network or a medical app on a smartphone to assist clinical decision-making. Regardless of the environmental circumstances, geographical barriers, and conventional infrastructures, it can share timely information between patients and health personnel, replacing the traditional face-to-face platform of medical care. Nevertheless, to not harm patients, it is pivotal that scientific accuracy, patient safety, and user privacy of mHealth apps be assured [18].

One systematic review that critically appraised $\mathrm{PCa}$ risk calculator apps maintained that the Rotterdam Prostate Cancer Risk Calculator and Coral-Prostate Cancer Nomogram Calculator outperformed other apps [3]. The authors utilized the validated user version of the Mobile Application Rating Scale to individually assess and rate 7 apps, including 3 categories of app quality ratings, subjective quality, and perceived impact [19]. Objective characteristics were thoroughly documented and assessed. None of these apps allowed confidentiality, data storage developing trends, or customization. Both the Rotterdam and Coral apps were found to help differentiate low-grade from high-grade $\mathrm{PCa}$, a noteworthy characteristic of patient counseling in active surveillance compared to other curative alternatives.

Mobile technology enables clinicians and patients to download the Rotterdam and Coral apps readily, and the owners can utilize these apps without an internet connection. They both have the advantages of being less time-consuming and more cost-effective, delivering better immediacy, upgradability, and shareability than the original risk calculators. In addition, they are globally available and recommended by the American
Urological Association and EAU guidelines to improve prediction and help determine the risk of PCa stratification. Compared with the Coral app, the conspicuous disadvantage of the Rotterdam app is its cost (\$1.99 USD). However, it has been proven to reach a wider audience, with availability on both Apple and Android platforms. While the Coral app has merely 1 language choice, the Rotterdam app has 7 different language options, including Chinese, Dutch, English, Estonian, German, Portuguese, and Spanish.

\section{Limitations}

This study was a single-institution retrospective study, and more cohorts from other Taiwanese hospitals are required to confirm the results. Also, the sample size in the prostatectomy cohort was rather small $(n=137)$. Notwithstanding that, this is the first study validating PCa risk calculators with postprostatectomy pathological outcomes, although the predictive accuracy of both apps for true high-grade PCa could not be completely determined. Further, no PHI data are available in our institution. The PHI is a combination of 3 blood tests measuring different forms of the PSA protein (total PSA, free PSA, and p2PSA) and calculated as $(\mathrm{p} 2 \mathrm{PSA} / \mathrm{fPSA}) \times \sqrt{ }$ tPSA. It is one of the predictors listed in the Rotterdam app, although the risks of $\mathrm{PCa}$ can still be calculated without PHI data. Few patients underwent mpMRI before biopsy in our cohort; however, mpMRI has emerged as an important prediction tool to identify clinically significant $\mathrm{PCa}$, especially before a repeated biopsy, which has been recommended by the guidelines [14,15]. The Rotterdam app and web-based ERSPC-RC is one of the PCa risk prediction models incorporating mpMRI (PI-RADS 1-5) [20]; nevertheless, the predictor of the PI-RADS used in the Rotterdam app was the first version, and most of our MRI images were graded according to the PI-RADS v2 guidelines. Moreover, the issues of interobserver variability and heterogeneous definitions of abnormality in mpMRI interpretation remain to be explored, and these MRI risk prediction models need to be validated further.

\section{Conclusions}

In our external validation study, the Rotterdam and Coral mHealth apps could be applied to the Taiwanese cohort of patients. Following an assessment of calibration, discrimination, and clinical utility, the Rotterdam app outperformed the Coral app for predicting both any-grade PCa and high-grade PCa. The size of the prostatectomy cohort was small; however, both mobile phone apps, to some extent, demonstrated a predictive capacity for true high-grade $\mathrm{PCa}$, confirmed by the whole prostate specimen. As of yet, there is no PCa risk calculator app developed specifically for the Taiwanese population; however, the Rotterdam app might be a good alternative to enhance the predictive accuracy of current methods for detecting PCa and high-grade PCa.

\section{Conflicts of Interest}

None declared.

\section{References}


1. Roobol MJ, Verbeek JF, van der Kwast T, Kümmerlin IP, Kweldam CF, van Leenders GJ. Improving the Rotterdam European Randomized Study of Screening for Prostate Cancer Risk Calculator for Initial Prostate Biopsy by Incorporating the 2014 International Society of Urological Pathology Gleason Grading and Cribriform growth. European Urology 2017 Jul;72(1):45-51. [doi: 10.1016/j.eururo.2017.01.033]

2. Ankerst DP, Hoefler J, Bock S, Goodman PJ, Vickers A, Hernandez J, et al. Prostate Cancer Prevention Trial Risk Calculator 2.0 for the Prediction of Low- vs High-grade Prostate Cancer. Urology 2014 Jun;83(6):1362-1368. [doi: 10.1016/j.urology.2014.02.035]

3. Adam A, Hellig JC, Perera M, Bolton D, Lawrentschuk N. 'Prostate Cancer Risk Calculator' mobile applications (Apps): a systematic review and scoring using the validated user version of the Mobile Application Rating Scale (uMARS). World J Urol 2017 Dec 8;36(4):565-573. [doi: 10.1007/s00345-017-2150-1]

4. De Nunzio C, Lombardo R, Tema G, Cancrini F, Russo GI, Chacon R, et al. Mobile phone apps for the prediction of prostate cancer: External validation of the Coral and Rotterdam apps. European Journal of Surgical Oncology 2019 Mar;45(3):471-476. [doi: 10.1016/j.ejso.2018.09.018]

5. Hung C, Yang C, Ou Y. Urologic cancer in Taiwan. Jpn. J. Clin. Oncol 2016 Apr 06;46(7):605-609. [doi: 10.1093/jijco/hyw038]

6. $\mathrm{Pu}$ Y. Prostate cancer in Taiwan: epidemiology and risk factors. Int J Androl 2000 Apr;23(S2):34-36. [doi: 10.1046/j.1365-2605.2000.00012.x]

7. Weinreb JC, Barentsz JO, Choyke PL, Cornud F, Haider MA, Macura KJ, et al. PI-RADS Prostate Imaging - Reporting and Data System: 2015, Version 2. European Urology 2016 Jan;69(1):16-40. [doi: 10.1016/j.eururo.2015.08.052]

8. Pereira-Azevedo N, Osório L, Fraga A, Roobol MJ. Rotterdam Prostate Cancer Risk Calculator: Development and Usability Testing of the Mobile Phone App. JMIR Cancer 2017 Jan 06;3(1):e1. [doi: 10.2196/cancer.6750]

9. Steyerberg EW, Vickers AJ, Cook NR, Gerds T, Gonen M, Obuchowski N, et al. Assessing the Performance of Prediction Models. Epidemiology 2010;21(1):128-138. [doi: 10.1097/ede.0b013e3181c30fb2]

10. Poyet C, Nieboer D, Bhindi B, Kulkarni GS, Wiederkehr C, Wettstein MS, et al. Prostate cancer risk prediction using the novel versions of the European Randomised Study for Screening of Prostate Cancer (ERSPC) and Prostate Cancer Prevention Trial (PCPT) risk calculators: independent validation and comparison in a contemporary Europe. BJU Int 2015 Oct 05;117(3):401-408. [doi: 10.1111/bju.13314]

11. Hosmer DW, Lemesbow S. Goodness of fit tests for the multiple logistic regression model. Comm. in Stats - Theory \& Methods 1980;9(10):1043-1069. [doi: 10.1080/03610928008827941]

12. DeLong ER, DeLong DM, Clarke-Pearson DL. Comparing the Areas under Two or More Correlated Receiver Operating Characteristic Curves: A Nonparametric Approach. Biometrics 1988 Sep;44(3):837. [doi: 10.2307/2531595]

13. Van Calster B, Wynants L, Verbeek JF, Verbakel JY, Christodoulou E, Vickers AJ, et al. Reporting and Interpreting Decision Curve Analysis: A Guide for Investigators. European Urology 2018 Dec;74(6):796-804. [doi:

10.1016/j.eururo.2018.08.038]

14. National Comprehensive Cancer Network. Prostate Cancer Early Detection (Version 2.2019). 2019. URL: https://www. nccn.org/professionals/physician gls/pdf/prostate detection.pdf [accessed 2019-07-29]

15. Mottet N, Bellmunt J, Bolla M, Briers E, Cumberbatch MG, De Santis M, et al. EAU-ESTRO-SIOG Guidelines on Prostate Cancer. Part 1: Screening, Diagnosis, and Local Treatment with Curative Intent. European Urology 2017 Apr;71(4):618-629. [doi: 10.1016/j.eururo.2016.08.003]

16. Louie K, Seigneurin A, Cathcart P, Sasieni P. Do prostate cancer risk models improve the predictive accuracy of PSA screening? A meta-analysis. Annals of Oncology 2015 May;26(5):848-864. [doi: 10.1093/annonc/mdu525]

17. Verep S, Erdem S, Ozluk Y, Kilicaslan I, Sanli O, Ozcan F. The pathological upgrading after radical prostatectomy in low-risk prostate cancer patients who are eligible for active surveillance: How safe is it to depend on bioptic pathology? Prostate 2019 Jul 03:1523-1529. [doi: 10.1002/pros.23873]

18. Pereira-Azevedo NM, Venderbos LDF. eHealth and mHealth in prostate cancer detection and active surveillance. Transl. Androl. Urol 2018 Feb;7(1):170-181. [doi: 10.21037/tau.2017.12.22]

19. Stoyanov SR, Hides L, Kavanagh DJ, Wilson H. Development and Validation of the User Version of the Mobile Application Rating Scale (uMARS). JMIR Mhealth Uhealth 2016 Jun 10;4(2):e72. [doi: 10.2196/mhealth.5849]

20. Schoots IG, Roobol MJ. Multivariate risk prediction tools including MRI for individualized biopsy decision in prostate cancer diagnosis: current status and future directions. World J Urol 2019 Mar 13;38(3):517-529. [doi: $\underline{10.1007 / \mathrm{s} 00345-019-02707-9]}$

\section{Abbreviations}

AUC: area under the receiver operating characteristic curve

EAU: European Association of Urology

ERSPC-RC: European Randomized Study of Screening for Prostate Cancer risk calculator

DRE: digital rectal examination

mHealth: mobile health 
mpMRI: multiparametric magnetic resonance imaging

MRI: magnetic resonance imaging

PCa: prostate cancer

PCPT: North American Prostate Cancer Prevention Trial

PHI: Prostate Health Index

PI-RADS: Prostate Imaging Reporting and Data System

PSA: prostate-specific antigen

PV: prostate volume

ROC: receiver operating characteristic

TRUS: transrectal ultrasound

Edited by G Eysenbach; submitted 07.10.19; peer-reviewed by PH Lin, C Fernández, $R$ Wassersug; comments to author 13.06.20; revised version received 03.07.20; accepted 11.11.20; published 18.12.20

Please cite as:

Chen IHA, Chu CH, Lin JT, Tsai JY, Yu CC, Sridhar AN, Sooriakumaran P, Loureiro RCV, Chand M

Prostate Cancer Risk Calculator Apps in a Taiwanese Population Cohort: Validation Study

J Med Internet Res 2020;22(12):e16322

URL: http://www.jmir.org/2020/12/e16322/

doi: $\underline{10.2196 / 16322}$

PMID: 33337340

(CI-Hsuan Alan Chen, Chi-Hsiang Chu, Jen-Tai Lin, Jeng-Yu Tsai, Chia-Cheng Yu, Ashwin Narasimha Sridhar, Prasanna Sooriakumaran, Rui C V Loureiro, Manish Chand. Originally published in the Journal of Medical Internet Research (http://www.jmir.org), 18.12.2020. This is an open-access article distributed under the terms of the Creative Commons Attribution License (https://creativecommons.org/licenses/by/4.0/), which permits unrestricted use, distribution, and reproduction in any medium, provided the original work, first published in the Journal of Medical Internet Research, is properly cited. The complete bibliographic information, a link to the original publication on http://www.jmir.org/, as well as this copyright and license information must be included. 